\title{
Factors associated with parasite aggregation levels in fishes from Brazil
}

\author{
Fatores associados aos níveis de agregação parasitária em peixes do Brasil \\ Cristina Fernandes do Amarante ${ }^{1 *}$; Wagner de Souza Tassinari ${ }^{1,2}$; José Luis Luque ${ }^{1,3}$; Maria Julia Salim Pereira ${ }^{1}$ \\ ${ }^{1}$ Pós-graduação em Ciências Veterinárias, Instituto de Veterinária, Universidade Federal Rural do Rio de Janeiro - UFRRJ, \\ Seropédica, RJ, Brasil \\ ${ }^{2}$ Departamento de Matemática, Instituto de Ciências Exatas, Universidade Federal Rural do Rio de Janeiro - UFRRJ, \\ Seropédica, RJ, Brasil \\ ${ }^{3}$ Departamento de Parasitologia Animal, Instituto de Veterinária, Universidade Federal Rural do Rio de Janeiro - UFRRJ, \\ Seropédica, RJ, Brasil \\ Received January 17, 2015 \\ Accepted March 18, 2015

\begin{abstract}
The degree of parasite aggregation is determined by a number of factors that are not well understood. In total, 3,746 fish from 73 species and their parasites were analyzed to determine associations between the degree of aggregation by taxon and specific characteristics of the fish. A multiple linear regression (95\% confidence interval - CI) was used to show that in most taxa, the degree of parasite aggregation was significantly higher in freshwater fish and schooling fish but varied according to taxon in relation to gender, habitat and feeding habits. Parasite aggregations were also significantly increased in fish with a large number of parasitic larvae and greater body length in all evaluated taxa. Because the coefficients of determination of the models were lower than $60 \%$ for all taxa, other factors may be involved in parasite aggregation. Conversely, marked significance was observed for the tested variables in relation to taxon dependence, which indicates that additional studies should be performed for these factors using comprehensive databases with larger samples per parasite species.
\end{abstract}

Keywords: Epidemiology, parasite ecology, multiple regression analysis.

\section{Resumo}

O nível de agregação parasitária é determinado por um conjunto de fatores não muito bem elucidados. Foram analisados 3.746 peixes pertencentes a 73 espécies e seus respectivos parasitos, com o objetivo de verificar a associação entre o nível de agregação por táxon e algumas características dos peixes. Por meio da técnica de regressão linear múltipla (IC 95\%), observou-se que o nível de agregação de parasitos foi significativamente maior em peixes dulcícolas e formadores de cardume, na maioria dos táxons, mas variou conforme o táxon em relação ao sexo, o hábitat e o hábito alimentar. A agregação parasitária também aumentou significativamente em peixes com maior proporção de larvas parasitas e com maior comprimento do corpo em todos os táxons avaliados. Tendo em vista que os coeficientes de determinação dos modelos para todos os táxons foram inferiores a $60 \%$, outros fatores podem estar envolvidos na agregação de parasitos. Por outro lado, as variáveis testadas assumem importância diferenciada na dependência do táxon, sinalizando para a necessidade de se continuar pesquisando esses fatores em bancos de dados mais abrangentes e com amostras maiores por espécies de parasitos.

Palavras-chave: Epidemiologia, ecologia parasitária, análise de regressão múltipla.

\footnotetext{
${ }^{*}$ Corresponding author: Cristina Fernandes do Amarante. Universidade Federal Rural do Rio de Janeiro, BR-465, Km 7, s/n, CEP 23890-000,

Seropédica, RJ, Brasil.

e-mail: cristinaf.amarante@gmail.com
} 


\section{Introduction}

The aggregate spatial distribution pattern of parasites is a common characteristic among various types of hosts and metazoan parasites and is considered a law of parasite ecology (CROFTON, 1971; VON ZUBEN, 1997; POULIN, 2007a). This distribution pattern can be observed in practice, with many hosts harboring few or no parasites and few hosts harboring many parasites (SHAW \& DOBSON, 1995; POULIN, 2007b).

Because of the heterogeneous size of parasitic infrapopulations, parasite spacial distribution is significant in the epidemiology of parasitic diseases and is observed in two distribution patterns: homogeneous distribution, which is associated with the mortality and density of parasites and the mortality of hosts induced by the parasites; and aggregate distribution, which is associated with heterogeneous host susceptibility to infection, parasite reproduction in the host and different abilities of hosts to eliminate parasites through immune responses or other mechanisms (ANDERSON \& GORDON, 1982).

The inherent characteristics and habits of hosts can also contribute to different degrees of parasite aggregation. An approach to assessing aggregation processes in species of fish parasites with the potential for use as a discriminator of stocks or populations of hosts was developed by Lester (2012), who analyzed aggregations according to parasite species by determining the relationship between parameters of aggregation and aspects of parasitic biology, such as the number of hosts participating in the biological cycle.

Conversely, Poulin (2013) conducted a meta-analysis with data on 410 samples compiled from several published articles to explain possible variations in parasite aggregation. In this study, the author used a mixed-effects model to analyze the different degrees of aggregation between host samples, and the parasite species, host species and study of origin were included as random effect variables. Thus, the effect of systems or researchers was also considered. The parasite taxon, developmental stages (larval/adult) and host size were variables included as fixed effects.

The approach of Poulin (2013) was based on knowledge (from experimental evidence) that changes in the host size in the studied samples and differences between parasitic stages (young and old) and their relationship with parasite aggregation could be confirmed in natural samples. As a result, Poulin (2013) observed that only the host sample size variable, which was included in the model as a confounding variable, was significant and explained $8 \%$ of the variability in aggregation. The author also observed that the combination of parasite species, host species and study of origin explained nearly two-thirds of the variability that was not explained by the fixed effects. Although Poulin (2013) considered the idiosyncrasies of the systems or researchers by using the mixed-effects model, the characteristics of studies that utilize databases can also produce different results compared with studies developed with data generated by the research group itself in a single location.

Because the presence of a variable can change the effects of another variable when they are analyzed simultaneously (MEDRONHO et al., 2009), certain explanatory variables that are considered in descriptive studies on fish parasite ecology and have the potential to explain the aggregation of parasites must be tested simultaneously. The present study aimed to evaluate whether parasite aggregation in certain taxa of fish parasites is associated with characteristics of the host, such as gender, size, habitat, schooling, feeding habits, aquatic environment and parasite development stage. This approach is intended to add new information related to determinants of change in the degree of aggregation characteristic of parasitic systems.

\section{Materials and Methods}

\section{Data sources}

The data analyzed in the present study belong to a database consisting of 73 species of fish (Table 1), with 54 marine and 19 freshwater species and a total of 3,983 specimens. The hosts and their parasites were collected between 1991 and 2009 on the coast of the state of Rio de Janeiro $\left(21^{\circ}-23^{\circ} \mathrm{S}\right.$ and $\left.42^{\circ}-45^{\circ} \mathrm{W}\right)$ and in the Guandu River ( $\left.22^{\circ} 48^{\prime} 2^{\prime \prime} \mathrm{S}, 43^{\circ} 37^{\prime} 35^{\prime \prime} \mathrm{W}\right)$. Fish and their parasites were identified at the Laboratory of Fish Parasitology of the Department of Animal Parasitology of the Federal Rural University of Rio de Janeiro (Universidade Federal Rural do Rio de Janeiro - UFRRJ) using the same methodological criteria throughout the collection period as previously described by Luque et al. (2004) and Azevedo et al. (2011). Fish are classified in the database according to species, gender, schooling, aquatic environment, habitat, feeding habits and body length. Parasite abundance was classified according to the development phase as either larvae or adult. Because of immature gonads, the gender of 237 specimens could not be identified, and they were excluded from the present study, which analyzed 3,746 individuals.

In the present study, because of the small number of samples of certain species, the parasites were grouped at higher taxa as follows: Nematoda, Monogenea, Trematoda, Cestoda, Acanthocephala, Hirudinea and Crustacea. Parasites from two taxa, Myxozoa and Mollusca, were excluded because only two host species were parasitized by species from these groups.

\section{Variables studied}

The explanatory variables used in the study were the host's gender (male/female), schooling (yes/no), aquatic environment (marine/freshwater), habitat (benthic/benthopelagic/pelagic), feeding habits (carnivore/planktivore/omnivore), and size $(\mathrm{cm})$ and the parasite's development stage (larva/adult). The aggregation indices of higher taxa were considered the outcome variables.

\section{Statistical analysis}

The aggregation indices for taxa and parasitic development stages were calculated using the dispersion index by dividing the variance by the parasitic mean (VON ZUBEN, 1997). The dispersion index was chosen for the statistical analysis because it is the most accepted and widely used parameter (WILSON et al., 2002), 
Table 1. Fish species that compose the analyzed database.

\begin{tabular}{|c|c|c|c|c|}
\hline Species & Family & Number of hosts & Environment & Mean body size $(\mathrm{cm})$ \\
\hline Aluterus monoceros & Monacanthiidae & 39 & marine & 31.2 \\
\hline Anchoa marinii & Engraulidae & 95 & marine & 9.8 \\
\hline Anchoa tricolor & Engraulidae & 103 & marine & 11.1 \\
\hline Archosargus rhomboidalis & Sparidae & 29 & marine & 31.3 \\
\hline Astyanax bimaculatus & Characidae & 40 & freshwater & 9.9 \\
\hline Astyanax parabybae & Characidae & 40 & freshwater & 10.4 \\
\hline Astronotus ocellatus & Cichlidae & 35 & freshwater & 20.4 \\
\hline Balistes capriscus & Balistidae & 33 & marine & 35 \\
\hline Balistes vetula & Balistidae & 18 & marine & 47.8 \\
\hline Brervoortia aurea & Clupeidae & 42 & marine & 29.6 \\
\hline Caranx hippos & Carangidae & 60 & marine & 43.9 \\
\hline Caranx latus & Carangidae & 55 & marine & 33.3 \\
\hline Centropomus undecimalis & Centropomidae & 30 & marine & 35.2 \\
\hline Cephalopholis fulva & Serranidae & 30 & marine & 20.6 \\
\hline Cichla ocellaris & Cichlidae & 26 & freshwater & 26.4 \\
\hline Chaetodipterus faber & Ephippidae & 110 & marine & 27.7 \\
\hline Cynoscion guatucupa & Sciaenidae & 73 & marine & 32.6 \\
\hline Cyphocharax gilbert & Curimatidae & 60 & freshwater & 16.3 \\
\hline Dactylopterus volitans & Dactylopteridae & 78 & marine & 22.9 \\
\hline Diapterus rhombeus & Gerreidae & 32 & marine & 17.4 \\
\hline Euthynnus alletteratus & Scombridae & 17 & marine & 43.9 \\
\hline Genidens barbus & Ariidae & 63 & marine & 43.8 \\
\hline Geophagus brasiliensis & Cichlidae & 50 & freshwater & 15.5 \\
\hline Genypterus brasiliensis & Ophidiidae & 21 & marine & 42.7 \\
\hline Gymnothorax moringa & Muraenidae & 30 & marine & 70.4 \\
\hline Gymnotus carapo & Gymnotidae & 30 & freshwater & 36.5 \\
\hline Haemulon steindachneri & Clupeidae & 80 & marine & 19.6 \\
\hline Hoplosternum littorale & Callichthyidae & 100 & freshwater & 19.7 \\
\hline Harengula clupeola & Haemulidae & 35 & marine & 20 \\
\hline Hypostomus affinis & Loricariidae & 31 & freshwater & 27.8 \\
\hline Leporinus copelandii & Anostomidae & 30 & freshwater & 34.8 \\
\hline Leporinus conirostris & Anostomidae & 18 & freshwater & 36.6 \\
\hline Lophius gastrophysus & Lophiidae & 30 & marine & 41.9 \\
\hline Loricariichthys castaneus & Loricariidae & 32 & freshwater & 27.9 \\
\hline Macrodon ancylodon & Sciaenidae & 31 & marine & 30 \\
\hline Menticirrhus americanus & Sciaenidae & 115 & marine & 28.4 \\
\hline Merluccius hubbsi & Merluccidae & 31 & marine & 38.5 \\
\hline Micropogonias furnieri & Sciaenidae & 100 & marine & 33.2 \\
\hline Mugil liza & Mugilidae & 34 & freshwater & 34.1 \\
\hline Mugil platanus & Mugilidae & 150 & marine & 54.8 \\
\hline Mullus argentinae & Mullidae & 100 & marine & 17.8 \\
\hline Mylossoma aureum & Characidae & 17 & freshwater & 15.7 \\
\hline Oligoplites palometa & Carangidae & 84 & marine & 38.2 \\
\hline Oligoplites saliens & Carangidae & 36 & marine & 36.8 \\
\hline Oligoplites saurus & Carangidae & 37 & marine & 29 \\
\hline Oligosarcus hepsetus & Characidae & 40 & freshwater & 16.6 \\
\hline Pagrus pagrus & Sparidae & 90 & marine & 29.5 \\
\hline Paralichthys isosceles & Paralichthyidae & 36 & marine & 31.2 \\
\hline Paralonchurus brasiliensis & Sciaenidae & 93 & marine & 21.1 \\
\hline Parona signata & Carangidae & 31 & marine & 37.5 \\
\hline Peprilus paru & Stromateidae & 81 & marine & 23.7 \\
\hline Percophis brasiliensis & Percophidae & 60 & marine & 43.6 \\
\hline
\end{tabular}


Table 1. Continued...

\begin{tabular}{|c|c|c|c|c|}
\hline Species & Family & Number of hosts & Environment & Mean body size $(\mathrm{cm})$ \\
\hline Pimelodus maculatus & Pimelodidae & 40 & freshwater & 23 \\
\hline Priacanthus arenatus & Priacanthidae & 58 & marine & 37.7 \\
\hline Pomatomus saltatrix & Pomatomidae & 55 & marine & 46.6 \\
\hline Prionotus punctatus & Triglidae & 80 & marine & 29.3 \\
\hline Pseudopercis numida & Pinguipedidae & 58 & marine & 47.7 \\
\hline Pseudopercis semifasciata & Pinguipedidae & 66 & marine & 38.7 \\
\hline Rhamdia quelen & Heptapteridae & 30 & freshwater & 32.7 \\
\hline Sarda sarda & Scombridae & 24 & marine & 45.4 \\
\hline Sardinella brasiliensis & Clupeidae & 35 & marine & 18.2 \\
\hline Sciadeichthys luniscutis & Ariidae & 69 & marine & 35.5 \\
\hline Scomber japonicus & Scombridae & 100 & marine & 25.8 \\
\hline Scomberomorus brasiliensis & Scombridae & 12 & marine & 46.4 \\
\hline Selene setapinnis & Carangidae & 53 & marine & 29.4 \\
\hline Sphyraena guachancho & Sphyraenidae & 36 & marine & 36.4 \\
\hline Tilapia rendalli & Cichlidae & 30 & freshwater & 22.1 \\
\hline Trachelyopterus striatulus & Auchenipteridae & 60 & freshwater & 19.2 \\
\hline Trichiurus lepturus & Trichiuridae & 55 & marine & 122.8 \\
\hline Tylosurus acus acus & Belonidae & 31 & marine & 72.5 \\
\hline Uraspis secunda & Carangidae & 21 & marine & 39.9 \\
\hline Urophycis brasiliensis & Phycidae & 26 & marine & 28.5 \\
\hline Urophycis mystacea & Phycidae & 46 & marine & 26.4 \\
\hline
\end{tabular}

although it is somewhat dependent on the parasite prevalence in large samples (POULIN, 2007b).

A univariate analysis was performed, consisting of calculating the mean aggregation indices for each species and the mean aggregation for each category of explanatory variable except for host size and larvae proportion.

Data normality was tested using the Shapiro-Wilk test. The non-parametric Wilcoxon test for unpaired samples and Kruskal-Wallis test were used to compare two or more than two independent groups, respectively (KATZ, 2006a). In this study, the groups were formed by the categories of explanatory variables. Pearson's linear correlation test was used to evaluate the correlation between the aggregation and proportion of larvae and the body length of the host. Subsequently, bivariate analyses were performed to better understand the behavior of the variables.

Thereafter, all of the variables were submitted to a multiple linear regression model fitting. The stepwise technique was used to select variables and obtain the most parsimonious model (final model) to explain variations in the degree of parasite aggregation (VENABLES \& RIPLEY, 2002; DOHOO et al., 2003; KATZ, 2006b).

The variables development stage and food type were included in the aggregation analysis only for the taxa whose cycles are indirect. For the analysis, the variable development stage was transformed into a proportion, and the proportion of larvae was used in the models.

Because of the low number of observations, the following categories were not analyzed in the Hirudinea taxon: pelagic (habitat variable) and planktivore (feeding habits variable).

All of the statistical calculations were performed with $\mathrm{R}$ software for Windows, version 2.15.2 (R DEVELOPMENT CORE TEAM, 2014) using a 5\% significance level.

\section{Results}

Table 2 shows that the three highest values for mean parasite aggregation were observed for the taxon Trematoda, followed by the taxa Nematoda and Cestoda. Female hosts exhibited greater aggregation than males except for in Hirudinea and Crustacea. Host species that live in schools also presented greater aggregation than species without this characteristic in all taxa. Parasites of marine fish presented greater aggregation in Nematoda, Trematoda, Acanthocephala, Hirudinea and Crustacea. Parasites of benthopelagic fish showed greater aggregation in Nematoda, Trematoda, Acantocephala, Cestoda, Hirudinea and Crustacea. Parasites of carnivorous hosts of the Trematoda, Cestoda and Hirudinea taxa showed greater aggregation compared with other taxa.

The degree of parasite aggregation had a positive and significant correlation with larval stage in the following taxa: Nematoda [0.08 (0.04; 0.11)], Acanthocephala $[0.08$ (0.04; 0.13)] and Cestoda $[0.28(0.25 ; 0.32)]$ and the adult stage in Trematoda $[0.14(0.11 ; 0.17)]$ and Cestoda $[0.29(0.25 ; 0.32)]$. Host size exhibited the same correlation type in the following taxa: Nematoda [0.18 $(0.14 ; 0.21)]$, Trematoda $[0.65(0.63 ; 0.67)]$, Acanthocephala $[0.23(0.19 ; 0.27)]$, Cestoda $[0.68(0.66 ; 0.70)]$ and Crustacea $[0.27(0.23 ; 0.30)]$.

A bivariate analysis (Table 3) was performed on the results of association tests and were later complemented by the multivariate analysis. Tables 4 and 5 show the results of the initial and final models. To fit the multiple linear regression models (Table 5), an increased degree of aggregation was associated with female hosts in the Monogena and Cestoda and with male hosts in the Crustacea taxon. In other taxa, the gender variable was not included in the final regression model and excluded because of a lack of significance. 
Table 2. Distribution of the means of the degrees of parasite aggregation in fish from the State of Rio de Janeiro.

\begin{tabular}{|c|c|c|c|c|c|c|c|c|}
\hline \multirow[t]{2}{*}{ Variables } & \multirow[t]{2}{*}{ Categories } & \multicolumn{7}{|c|}{$\begin{array}{l}\text { Total means by taxon. and means and their respective confidence intervals }(95 \%) \\
\text { by taxon according to the categories of explanatory variables. }\end{array}$} \\
\hline & & $\begin{array}{c}\text { Nematoda } \\
(29.10)\end{array}$ & $\begin{array}{c}\text { Monogenea } \\
(19.11)\end{array}$ & $\begin{array}{c}\text { Trematoda } \\
(63.84)\end{array}$ & $\begin{array}{c}\text { Acanthocephala } \\
(6.01)\end{array}$ & $\begin{array}{l}\text { Cestoda } \\
(23.06)\end{array}$ & $\begin{array}{l}\text { Hirudinea } \\
(2.20)\end{array}$ & $\begin{array}{c}\text { Crustacea } \\
(16.16)\end{array}$ \\
\hline \multirow[t]{2}{*}{ Sex } & Male & $\begin{array}{c}27.44^{*} \\
{[24.11 ; 30.77]}\end{array}$ & $\begin{array}{c}16.10 \\
{[14.75 ; 17.45]}\end{array}$ & $\begin{array}{c}40.18 \\
{[35.77 ; 44.59]}\end{array}$ & $\begin{array}{c}5.52^{*} \\
{[5.10 ; 5.94]}\end{array}$ & $\begin{array}{c}13.96^{*} \\
{[12.27 ; 15.63]}\end{array}$ & $\begin{array}{c}4.79 \\
{[4.03 ; 4.85]}\end{array}$ & $\begin{array}{c}16.41 \\
{[15.01 ; 17.79]}\end{array}$ \\
\hline & Female & $\begin{array}{c}30.12 \\
{[26.87 ; 33.35]}\end{array}$ & $\begin{array}{c}19.98 \\
{[18.24 ; 21.74]}\end{array}$ & $\begin{array}{c}71.72 \\
{[60.55 ; 82.89]}\end{array}$ & $\begin{array}{c}6.73 \\
{[6.29 ; 7.17]}\end{array}$ & $\begin{array}{c}29.50 \\
{[25.54 ; 33.44]}\end{array}$ & $\begin{array}{c}3.86 \\
{[3.30 ; 4.06]}\end{array}$ & $\begin{array}{c}16.11 \\
{[14.71 ; 17.49]}\end{array}$ \\
\hline \multirow[t]{2}{*}{ School } & Yes & $\begin{array}{c}33.39^{*} \\
{[30.46 ; 36.32]}\end{array}$ & $\begin{array}{c}18.51^{*} \\
{[17.21 ; 19.83]}\end{array}$ & $\begin{array}{c}66.43^{*} \\
{[59.94 ; 74.92]}\end{array}$ & $\begin{array}{c}6.65^{*} \\
{[6.28 ; 7.02]}\end{array}$ & $\begin{array}{c}23.07^{*} \\
{[20.3625 .76]}\end{array}$ & $\begin{array}{c}5.88^{*} \\
{[5.00 ; 5.74]}\end{array}$ & $\begin{array}{c}17.49^{*} \\
{[16.32 ; 18.64]}\end{array}$ \\
\hline & No & $\begin{array}{c}15.14 \\
{[11.67 ; 13.81]}\end{array}$ & $\begin{array}{c}15.99 \\
{[14.33 ; 16.67}\end{array}$ & $\begin{array}{c}13.39 \\
{[12.23 ; 14.53]}\end{array}$ & $\begin{array}{c}4.11 \\
{[3.94 ; 4.26]}\end{array}$ & $\begin{array}{c}18.25 \\
{[16.16 ; 20.34]}\end{array}$ & $\begin{array}{c}1.41 \\
{[1.36 ; 1.44]}\end{array}$ & $\begin{array}{c}12.01 \\
{[10.29 ; 13.71]}\end{array}$ \\
\hline \multirow[t]{2}{*}{ Environment } & Marine & $\begin{array}{c}33.55^{*} \\
{[30.73 ; 36.35]}\end{array}$ & $\begin{array}{c}16.58^{*} \\
{[15.35 ; 17.83]^{*}}\end{array}$ & $\begin{array}{c}64.32^{*} \\
{[56.97 ; 71.69]}\end{array}$ & $\begin{array}{c}6.43 \\
{[6.08 ; 6.78]}\end{array}$ & $\begin{array}{c}21.68 \\
{[19.21 ; 24.13]}\end{array}$ & $\begin{array}{c}4.94 \\
{[4.13 ; 4.87]}\end{array}$ & $\begin{array}{c}16.67^{*} \\
{[15.55 ; 17.79]}\end{array}$ \\
\hline & Freshwater & $\begin{array}{c}10.51 \\
{[7.71 ; 13.29]}\end{array}$ & $\begin{array}{c}27.01 \\
{[24.72 ; 29.32]}\end{array}$ & $\begin{array}{c}18.24 \\
{[15.88 ; 20.56]}\end{array}$ & $\begin{array}{c}3.42 \\
{[3.26 ; 3.58]}\end{array}$ & $\begin{array}{c}30.84 \\
{[27.54 ; 34.14]}\end{array}$ & $\begin{array}{c}3.18 \\
{[2.89 ; 3.43]}\end{array}$ & $\begin{array}{c}11.98 \\
{[10.20 ; 13.76]}\end{array}$ \\
\hline \multirow[t]{3}{*}{ Habitat } & Benthic & $\begin{array}{c}17.58 \\
{[15.80 ; 19.32]}\end{array}$ & $\begin{array}{c}26.55^{* *} \\
{[24.05 ; 29.09]}\end{array}$ & $\begin{array}{c}11.52^{* *} \\
{[11.05 ; 11.99]}\end{array}$ & $\begin{array}{c}2.45^{* *} \\
{[2.32 ; 2.58]}\end{array}$ & $\begin{array}{c}8.46^{* *} \\
{[7.55 ; 9.35]}\end{array}$ & $\begin{array}{c}3.22^{* *} \\
{[3.02 ; 3.42]}\end{array}$ & $\begin{array}{c}12.52^{* *} \\
{[11.00 ; 14.03]}\end{array}$ \\
\hline & $\begin{array}{l}\text { Benthic } \\
\text { pelagic }\end{array}$ & $\begin{array}{c}38.01 \\
{[33.24 ; 42.76]}\end{array}$ & $\begin{array}{c}15.45 \\
{[14.12 ; 16.78]}\end{array}$ & $\begin{array}{c}92.25 \\
{[78.54 ; 105.94]}\end{array}$ & $\begin{array}{c}11.80 \\
{[11.14 ; 12.44]}\end{array}$ & $\begin{array}{c}41.92 \\
{[37.00 ; 46.82]}\end{array}$ & $\begin{array}{c}5.52 \\
{[4.91 ; 6.11]}\end{array}$ & $\begin{array}{c}24.48 \\
{[22.69 ; 26.27]}\end{array}$ \\
\hline & Pelagic & $\begin{array}{c}29.28 \\
{[25.31 ; 33.25]}\end{array}$ & $\begin{array}{c}10.52 \\
{[9.81 ; 11.21]}\end{array}$ & $\begin{array}{c}68.49 \\
{[63.72 ; 73.28]}\end{array}$ & $\begin{array}{c}3.75 \\
{[3.47 ; 4.00]}\end{array}$ & $\begin{array}{c}7.26 \\
{[6.57 ; 7.93]}\end{array}$ & $\dagger$ & $\begin{array}{c}6.68 \\
{[5.99 ; 7.37]}\end{array}$ \\
\hline \multirow[t]{3}{*}{$\begin{array}{l}\text { Feeding } \\
\text { habits }\end{array}$} & Carnivorous & $\begin{array}{c}28.86^{* *} \\
{[26.41 ; 31.29]}\end{array}$ & $\begin{array}{c}7.51^{* *} \\
{[7.05 ; 7.97]}\end{array}$ & $\begin{array}{c}133.90^{* *} \\
{[110.51 ; 157.29]}\end{array}$ & $\begin{array}{c}4.73^{* *} \\
{[4.42 ; 5.00]}\end{array}$ & $\begin{array}{c}50.20^{* *} \\
{[42.82 ; 57.58]}\end{array}$ & $\begin{array}{c}12.20^{* *} \\
{[10.97 ; 13.45]}\end{array}$ & $\begin{array}{c}12.30^{* *} \\
{[10.79 ; 13.79]}\end{array}$ \\
\hline & Planktivorous & $\begin{array}{c}1.93 \\
{[1.88 ; 1.98]}\end{array}$ & $\begin{array}{c}1.95 \\
{[1.80 ; 2.0]}\end{array}$ & $\begin{array}{c}65.17 \\
{[55.90 ; 74.44]}\end{array}$ & $\begin{array}{c}1.01 \\
{[1.00 ; 1.02]}\end{array}$ & $\begin{array}{c}1.50 \\
{[1.48 ; 1.48]}\end{array}$ & $\dagger$ & $\begin{array}{c}14.92 \\
{[13.33 ; 16.55]}\end{array}$ \\
\hline & Omnivorous & $\begin{array}{c}31.57 \\
{[28.26 ; 34.84]}\end{array}$ & $\begin{array}{c}22.74 \\
{[21.22 ; 24.28]}\end{array}$ & $\begin{array}{c}26.49 \\
{[24.87 ; 28.11]}\end{array}$ & $\begin{array}{c}7.10 \\
{[6.68 ; 7.52]}\end{array}$ & $\begin{array}{c}8.60 \\
{[7.97 ; 9.19]}\end{array}$ & $\begin{array}{c}2.65 \\
{[2.55 ; 2.71]}\end{array}$ & $\begin{array}{c}17.93 \\
{[16.60 ; 19.24]}\end{array}$ \\
\hline
\end{tabular}

$\left(^{*}\right)$ Significant Wilcoxon Test. $\left(^{* *}\right)$ Significant Kruskal-Wallis Test. (†) Excluded category.

Table 3. Bivariate analysis of the parasite aggregation in fish from the State of Rio de Janeiro.

\begin{tabular}{|c|c|c|c|c|c|c|c|}
\hline \multirow{2}{*}{ Variables } & \multicolumn{7}{|c|}{ Regression coefficients and their respective confidence intervals $(95 \%)$. according to the taxa } \\
\hline & Nematoda & Monogenea & Trematoda & Acanthocephala & Cestoda & Hirudinea & Crustacea \\
\hline Sex (Ref. Male) & $\begin{array}{l}2.67 \\
{[-2.26 ; 7.61]}\end{array}$ & $\begin{array}{l}3.88^{*} \\
\quad[1.40 ; 6.37]\end{array}$ & $\begin{array}{l}31.54^{*} \\
{[19.40 ; 43.68]}\end{array}$ & $\begin{array}{l}1.22^{*} \\
{[0.40 ; 2.04]}\end{array}$ & $\begin{array}{l}15.54^{*} \\
{[10.32 ; 20.76]}\end{array}$ & $\begin{array}{l}-0.93 \\
\quad[-1.91 ; 0.04]\end{array}$ & $\begin{array}{l}-0.30 \\
{[-2.54 ; 1.94]}\end{array}$ \\
\hline School (Ref. Yes) & $\begin{array}{l}-20.64^{*} \\
{[-26.52 ;-14.75]}\end{array}$ & $\begin{array}{l}-2.52 \\
\quad[-5.72 ; 0.68]\end{array}$ & $\begin{array}{l}-54.04^{*} \\
{[-68.76 ;-39.31]}\end{array}$ & $\begin{array}{l}-2.53^{*} \\
\quad[-3.57 ;-1.50]\end{array}$ & $\begin{array}{l}-4.82 \\
{[-11.18 ; 1.54]}\end{array}$ & $\begin{array}{l}-4.47^{*} \\
{[-5.46 ;-3.47]}\end{array}$ & $\begin{array}{l}-5.48^{*} \\
\quad[-8.15 ;-2.80]\end{array}$ \\
\hline $\begin{array}{l}\text { Environment } \\
\text { (Ref. Marine) }\end{array}$ & $\begin{array}{l}23.05^{*} \\
{[-29.09 ;-17.00]}\end{array}$ & $\begin{array}{l}10.42^{*} \\
\quad[6.86 ; 14.00]\end{array}$ & $\begin{array}{l}-46.09^{*} \\
{[-61.72 ;-30.45]}\end{array}$ & $\begin{array}{l}-3.01^{*} \\
\quad[-4.44 ;-1.59]\end{array}$ & $\begin{array}{l}9.15 \\
{[-4.69 ; 23.00]}\end{array}$ & $\begin{array}{l}-1.77^{*} \\
\quad[-2.79 ;-0.75]\end{array}$ & $\begin{array}{l}-4.69^{*} \\
\quad[-8.62 ;-0.77]\end{array}$ \\
\hline \multicolumn{8}{|l|}{$\begin{array}{l}\text { Habitat } \\
\text { (Ref. Benthic) }\end{array}$} \\
\hline Benthic pelagic & $\begin{array}{l}20.43^{*} \\
{[14.93 ; 25.93]}\end{array}$ & $\begin{array}{l}-11.10^{*} \\
\quad[-13.89 ;-8.32]\end{array}$ & $\begin{array}{l}80.73^{*} \\
{[67.32 ; 94.13]}\end{array}$ & $\begin{array}{l}9.35^{*} \\
{[8.52 ; 10.17]}\end{array}$ & $\begin{array}{l}33.46^{*} \\
\quad[27.67 ; 39.25]\end{array}$ & $\begin{array}{l}2.29^{*} \\
\quad[1.33 ; 3.26]\end{array}$ & $\begin{array}{l}11.96^{*} \\
\quad[9.49 ; 14.43]\end{array}$ \\
\hline Pelagic & $\begin{array}{l}11.70^{*} \\
\quad[4.77 ; 18.63]\end{array}$ & $\begin{array}{l}-16.03^{*} \\
{[-19.57 ;-12.49]}\end{array}$ & $\begin{array}{l}56.96^{*} \\
{[40.43 ; 73.49]}\end{array}$ & $\begin{array}{l}1.30^{*} \\
\quad[0.32 ; 2.28]\end{array}$ & $\begin{array}{c}-1.20 \\
{[-8.14 ; 5.74]}\end{array}$ & $\dagger$ & $\begin{array}{l}-5.84^{*} \\
\quad[-8.78 ;-2.91]\end{array}$ \\
\hline \multicolumn{8}{|l|}{$\begin{array}{l}\text { Feeding habits } \\
\text { (Ref. carnivorous) }\end{array}$} \\
\hline Planktivorous & $\begin{array}{l}-26.93^{*} \\
{[-37.39 ;-16.47]}\end{array}$ & \# & $\begin{array}{l}-68.77^{*} \\
{[-93.46 ;-44.08]}\end{array}$ & $\begin{array}{l}-3.72^{*} \\
\quad[-5.68 ;-1.76]\end{array}$ & $\begin{array}{l}-48.70^{*} \\
{[-61.99 ;-35.41]}\end{array}$ & $\dagger$ & \# \\
\hline Omnivorous & $\begin{array}{c}2.71 \\
{[-3.00 ; 8.42]}\end{array}$ & \# & $\begin{array}{l}-107.45^{*} \\
{[-121.35 ;-93.55]}\end{array}$ & $\begin{array}{l}2.37^{*} \\
\quad[1.44 ; 3.29]\end{array}$ & $\begin{array}{l}-41.60^{*} \\
{[-47.00 ;-36.20]}\end{array}$ & \# & \# \\
\hline Body length & $\begin{array}{l}0.70^{*} \\
\quad[0.56 ; 0.83]\end{array}$ & $\begin{array}{l}-0.05 \\
\quad[-0.12 ; 0.01]\end{array}$ & $\begin{array}{l}6.83^{*} \\
{[6.57 ; 7.09]}\end{array}$ & $\begin{array}{l}0.11^{*} \\
\quad[0.09 ; 0.13]\end{array}$ & $\begin{array}{l}2.46^{*} \\
\quad[2.35 ; 2.56]\end{array}$ & $\begin{array}{l}0.17^{*} \\
\quad[0.12 ; 0.21]\end{array}$ & $\begin{array}{l}0.44^{*} \\
\quad[0.38 ; 0.50]\end{array}$ \\
\hline Larval stage & $\begin{array}{l}13.25^{*} \\
\quad[7.54 ; 18.96]\end{array}$ & \# & $\begin{array}{l}-0.22 \\
{[-22.47 ; 22.02]}\end{array}$ & $\begin{array}{l}2.44^{*} \\
\quad[1.16 ; 3.71]\end{array}$ & $\begin{array}{l}44.91^{*} \\
{[39.04 ; 50.78]}\end{array}$ & $\#$ & \# \\
\hline
\end{tabular}

$\left(^{*}\right)$ p-value $<5 \%$. (†) Category not analized. (\#) Variable not analized. 
Table 4. Initial model of the multivariate linear regression analysis of parasite aggregation in fish from the State of Rio de Janeiro.

\begin{tabular}{|c|c|c|c|c|c|c|c|c|}
\hline \multirow{2}{*}{$\begin{array}{c}\text { Variables/ } \\
\text { metric }\end{array}$} & \multicolumn{8}{|c|}{ Regression coefficients and their respective confidence intervals $(95 \%)$ according to the taxa } \\
\hline & Categories & Nematoda & Monogenea & Trematoda & Acanthocephala & Cestoda & Hirudinea & Crustacea \\
\hline $\begin{array}{l}\text { Sex } \\
\text { (Ref. Male) }\end{array}$ & & $\begin{array}{l}-1.43 \\
{[-6.29 ; 3.44]}\end{array}$ & $\begin{array}{l}3.83 \\
{[1.38 ; 6.28]}\end{array}$ & $\begin{array}{c}4.00 \\
{[-4.54 ; 12.54]}\end{array}$ & $\begin{array}{l}0.37 \\
{[-0.33 ; 1.08]}\end{array}$ & $\begin{array}{l}4.93 \\
{[1.33 ; 8.53]}\end{array}$ & $\begin{array}{l}-0.22 \\
{[-1.14 ; 0.71]}\end{array}$ & $\begin{array}{l}-2.69 \\
{[-4.76 ;-0.63]}\end{array}$ \\
\hline $\begin{array}{l}\text { School (Ref. } \\
\text { yes) }\end{array}$ & & $\begin{array}{l}-13.82 \\
{[-20.33 ; 7.31]}\end{array}$ & $\begin{array}{l}-8.83 \\
{[-12.44 ;-5.21]}\end{array}$ & $\begin{array}{l}-93.42 \\
{[-104.96 ;-81.88]}\end{array}$ & $\begin{array}{l}1.61 \\
{[0.65 ; 2.58]}\end{array}$ & $\begin{array}{c}-34.88 \\
{[-39.81 ;-29.95]}\end{array}$ & $\begin{array}{l}-6.75 \\
{[-7.82 ;-5.67]}\end{array}$ & $\begin{array}{l}-14.16 \\
{[-16.99 ;-11.33]}\end{array}$ \\
\hline $\begin{array}{l}\text { Environment } \\
\text { (Ref. marine) }\end{array}$ & & $\begin{array}{l}-16.84 \\
{[-23.66 ;-10.03]}\end{array}$ & $\begin{array}{l}11.87 \\
{[8.00 ; 15.75]}\end{array}$ & $\begin{array}{l}72.36 \\
{[60.48 ; 84.24]}\end{array}$ & $\begin{array}{l}-8.27 \\
{[-9.61 ;-6.92]}\end{array}$ & $\begin{array}{l}30.21 \\
{[20.70 ; 39.71]}\end{array}$ & $\begin{array}{l}1.61 \\
{[0.50 ; 2.72]}\end{array}$ & $\begin{array}{l}-2.4 \\
{[-6.21 ; 1.40]}\end{array}$ \\
\hline \multirow[t]{2}{*}{$\begin{array}{l}\text { Habitat } \\
\text { (Ref. benthic) }\end{array}$} & $\begin{array}{l}\text { Benthic } \\
\text { pelagic }\end{array}$ & $\begin{array}{l}13.16 \\
{[7.23 ; 19.09]}\end{array}$ & $\begin{array}{l}-13.36 \\
{[-16.55 ;-10.18]}\end{array}$ & $\begin{array}{l}-16.99 \\
{[-27.26 ;-6.72]}\end{array}$ & $\begin{array}{l}11.56 \\
{[10.64 ; 12.48]}\end{array}$ & $\begin{array}{l}-0.39 \\
{[-4.76 ; 3.98]}\end{array}$ & $\begin{array}{l}-0.68 \\
{[-1.70 ; 0.33]}\end{array}$ & $\begin{array}{l}3.19 \\
{[0.57 ; 5.82]}\end{array}$ \\
\hline & Pelagic & $\begin{array}{l}32.57 \\
{[22.57 ; 42.57]}\end{array}$ & $\begin{array}{l}-3.33 \\
{[-8.80 ; 2.14]}\end{array}$ & $\begin{array}{l}-21.88 \\
{[-39.46 ;-4.29]}\end{array}$ & $\begin{array}{l}6.52 \\
{[5.12 ; 7.92]}\end{array}$ & $\begin{array}{l}-41.66 \\
{[-48.33 ;-34.99]}\end{array}$ & $\dagger$ & $\begin{array}{l}-10.02 \\
{[-12.94 ;-7.11]}\end{array}$ \\
\hline \multirow{2}{*}{$\begin{array}{l}\text { Feeding habits } \\
\text { (Ref. } \\
\text { carnivorous) }\end{array}$} & Planktivorous & $\begin{array}{l}-20.73 \\
{[-32.82 ;-8.64]}\end{array}$ & \# & $\begin{array}{l}168.97 \\
{[147.62 ; 190.31]}\end{array}$ & $\begin{array}{l}-1.36 \\
{[-3.19 ; 0.46]}\end{array}$ & $\begin{array}{l}44.93 \\
{[34.70 ; 55.15]}\end{array}$ & $\dagger$ & \# \\
\hline & Omnivorous & $\begin{array}{l}27.57 \\
{[19.84 ; 35.30]}\end{array}$ & \# & $\begin{array}{l}-4.28 \\
{[-18.28 ; 9.72]}\end{array}$ & $\begin{array}{l}5.99 \\
{[4.85 ; 7.13]}\end{array}$ & $\begin{array}{c}31.60 \\
{[-37.04 ;-26.15]}\end{array}$ & \# & \# \\
\hline Body size $(\mathrm{cm})$ & & $\begin{array}{l}0.69 \\
{[0.52 ; 0.86]}\end{array}$ & $\begin{array}{l}0.15 \\
{[0.07 ; 0.23]}\end{array}$ & $\begin{array}{l}8.39 \\
{[8.09 ; 8.69]}\end{array}$ & $\begin{array}{l}0.03 \\
{[0.01 ; 0.06]}\end{array}$ & $\begin{array}{l}2.23 \\
{[2.11 ; 2.34]}\end{array}$ & $\begin{array}{l}0.30 \\
{[0.24 ; 0.35]}\end{array}$ & $\begin{array}{l}0.34 \\
{[0.28 ; 0.40]}\end{array}$ \\
\hline Larval stage & & $\begin{array}{l}7.19 \\
{[1.07 ; 13.31]}\end{array}$ & \# & $\begin{array}{l}49.29 \\
{[33.21 ; 65.38]}\end{array}$ & $\begin{array}{l}2.58 \\
{[1.46 ; 3.70]}\end{array}$ & $\begin{array}{l}25.14 \\
{[20.74 ; 29.54]}\end{array}$ & \# & \# \\
\hline $\mathbf{R}^{2}$ ajusted & & $8 \%$ & $7 \%$ & $53 \%$ & $32 \%$ & $56 \%$ & $14 \%$ & $17 \%$ \\
\hline
\end{tabular}

(†) Category not analized. (\#) Variable not analized.

Table 5. Final model of the multivariate linear regression analysis of parasite aggregation in fish from the State of Rio de Janeiro.

\begin{tabular}{|c|c|c|c|c|c|c|c|c|}
\hline \multicolumn{2}{|c|}{ Variables/metric } & \multicolumn{7}{|c|}{ Regression coefficients and their respective confidence intervals $(95 \%)$ according to the taxa } \\
\hline & Categories & Nematoda & Monogenea & Trematoda & Acanthocephala & Cestoda & Hirudinea & Crustacea \\
\hline $\begin{array}{l}\text { Sex } \\
\text { (Ref. Male) }\end{array}$ & & - & $\begin{array}{l}4.00 \\
{[1.53 ; 6.47]}\end{array}$ & ( & - & $\begin{array}{l}4.93 \\
{[1.33 ; 8.53]}\end{array}$ & ( & $\begin{array}{l}-2.64 \\
{[-4.70 ;-0.57]}\end{array}$ \\
\hline $\begin{array}{l}\text { School } \\
\text { (Ref. Yes) }\end{array}$ & & $\begin{array}{l}-13.74 \\
{[-20.24 ;-7.23]}\end{array}$ & $\begin{array}{l}-12.76 \\
{[-16.21 ;-9.32]}\end{array}$ & $\begin{array}{l}-93.13 \\
{[-104.65 ;-81.60]}\end{array}$ & $\begin{array}{l}1.77 \\
{[0.77 ; 2.76]}\end{array}$ & $\begin{array}{l}-34.88 \\
{[-39.81 ;-29.95]}\end{array}$ & $\begin{array}{l}-6.57 \\
{[-7.60 ;-5.55]}\end{array}$ & $\begin{array}{l}-14.49 \\
{[-17.28 ;-11.69]}\end{array}$ \\
\hline $\begin{array}{l}\text { Environment } \\
\text { (Ref. marine) }\end{array}$ & & $\begin{array}{l}19.17 \\
{[-23.63 ;-12.71]}\end{array}$ & $\begin{array}{l}14.60 \\
{[10.76 ; 18.43]}\end{array}$ & $\begin{array}{l}71.98 \\
{[60.14 ; 83.83]}\end{array}$ & $\begin{array}{l}-8.17 \\
{[-9.52 ;-6.82]}\end{array}$ & $\begin{array}{l}30.21 \\
{[20.70 ; 39.71]}\end{array}$ & $\begin{array}{l}1.68 \\
{[0.58 ; 2.77]}\end{array}$ & - \\
\hline \multirow[t]{2}{*}{$\begin{array}{l}\text { Habitat } \\
\text { Ref.benthic) }\end{array}$} & Benthic pelagic & $\begin{array}{l}12.03 \\
{[6.19 ; 17.86]}\end{array}$ & $\begin{array}{l}-16.50 \\
{[-19.59 ;-13.41]}\end{array}$ & $\begin{array}{l}-17.15 \\
{[-27.41 ;-6.89]}\end{array}$ & $\begin{array}{l}11.40 \\
{[10.48 ; 12.32]}\end{array}$ & $\begin{array}{l}-0.39 \\
{[-4.76 ; 3.98]}\end{array}$ & - & $\begin{array}{l}2.78 \\
{[0.24 ; 5.32]}\end{array}$ \\
\hline & Pelagic & $\begin{array}{l}30.82 \\
{[20.95 ; 40.68]}\end{array}$ & $\begin{array}{l}-18.29 \\
{[-21.97 ;-14.60]}\end{array}$ & $\begin{array}{l}-22.18 \\
{[-39.75 ;-4.61]}\end{array}$ & $\begin{array}{l}7.09 \\
{[5.69 ; 8.49]}\end{array}$ & $\begin{array}{l}-41.66 \\
{[-48.33 ;-34.99]}\end{array}$ & $\dagger$ & $\begin{array}{l}-9.96 \\
{[-12.88 ;-7.05]}\end{array}$ \\
\hline \multirow{2}{*}{$\begin{array}{l}\text { Feeding habits } \\
\text { (Ref. } \\
\text { carnivorous) }\end{array}$} & Planktivorous & $\begin{array}{l}-21.52 \\
{[-33.60 ;-9.45]}\end{array}$ & \# & $\begin{array}{l}169.32 \\
{[147.99 ; 190.65]}\end{array}$ & $\begin{array}{l}-1.67 \\
{[-3.50 ; 0.16]}\end{array}$ & $\begin{array}{l}44.93 \\
{[34.70 ; 55.15]}\end{array}$ & $\dagger$ & \# \\
\hline & Omnivorous & $\begin{array}{l}27.80 \\
{[20.09 ; 35.51]}\end{array}$ & \# & $\begin{array}{l}-3.92 \\
{[-17.90 ; 10.06]}\end{array}$ & $\begin{array}{l}6.31 \\
{[5.17 ; 7.44]}\end{array}$ & $\begin{array}{l}-31.60 \\
{[-37.04-26.15]}\end{array}$ & \# & \# \\
\hline $\begin{array}{l}\text { Body size } \\
\text { (cm) }\end{array}$ & & $\begin{array}{l}0.70 \\
{[0.54 ; 0.87]}\end{array}$ & $\begin{array}{l}0.09 \\
\quad[0.01 ; 0.16]\end{array}$ & $\begin{array}{l}8.41 \\
\quad[8.11 ; 8.71]\end{array}$ & $\begin{array}{l}0.05 \\
\quad[0.03 ; 0.07]\end{array}$ & $\begin{array}{l}2.23 \\
{[2.11 ; 2.34]}\end{array}$ & $\begin{array}{l}0.28 \\
{[0.23 ; 0.33]}\end{array}$ & $\begin{array}{l}0.35 \\
{[0.29 ; 0.41]}\end{array}$ \\
\hline Larval stage & & te & \# & $\begin{array}{l}49.54 \\
{[33.47 ; 65.62]}\end{array}$ & $\begin{array}{l}1.36 \\
{[-2.69 ;-0.04]}\end{array}$ & $\begin{array}{l}25.14 \\
{[20.74 ; 29.54]}\end{array}$ & \# & $\#$ \\
\hline$R^{2}$ ajusted & & $8 \%$ & $6 \%$ & $53 \%$ & $31 \%$ & $56 \%$ & $14 \%$ & $17 \%$ \\
\hline
\end{tabular}

(†) Category not analized. (-) Variable excluded. (\#) variable not analized.

Freshwater schooling host species significantly influenced the increased parasite aggregation in most taxa. Parasite aggregation was higher in the Monogenea, Trematoda and Cestoda in benthic host species, Acanthocephala and Crustacea in benthopelagic species and Nematoda in pelagic species. In the Nematoda and Acanthocephala, the increased parasitic aggregation was significantly associated with omnivorous fish species, whereas in the Cestoda and Trematoda, it was associated with planktivorous species. The larval development stage remained in the final model in the
Trematoda, Acanthocephala and Cestoda and contributed to increased parasite aggregation; however, this parameter was only calculated for species that develop in an indirect cycle.

\section{Discussion}

Studies on the degree of parasite aggregation should use a comparative approach to obtain results that provide a better explanation of the factors that determine this characteristic of 
parasitic populations. Fish are considered a good model for studies on the aggregate distribution of parasites as well as other types of ecological aspects because they are easily obtained and consequently provide sufficient samples for statistical analyses (LUQUE et al., 2013).

Studies on the structure of parasitic fish communities in Brazil have provided results on parasite aggregation (LUQUE et al., 1996; ISAAC et al., 2000; LUQUE \& ALVES, 2001; LUQUE et al., 2008); however, they have not tested the association of parasite aggregation with any biological characteristics of the hosts, which drastically decreases the possibility of extracting patterns that might contribute to explaining the variability of aggregation. Furthermore, this information is included in studies that describe parasitic communities with no comparative purpose.

In the present study, problems detected in previous analyses were minimized. Such problems were attributed to databases that were compiled from different sources and used in these analyses (LUQUE et al., 2004). Differences in the methods used to detect and identify parasites in different sources may cause reading variations in the results. Furthermore, different host species included in the same data set often are originated from different geographical areas. The availability of different parasite species has a strong regional influence because of variation between regions, which adds another element of fluctuation to these datasets. Therefore, the consistency of the results of the present study was improved because the dataset included fish from the same geographical location, and all of the hosts and parasites were examined and identified using the same standards of a single research group. In addition, the present study tested the significance of biotic variables as determinants of the degree of parasite aggregation, which had not been tested to date.

The results of the bivariate and multivariate analyses are complementary and demonstrate the active strength of one variable acting on another when they are analyzed simultaneously, which can change the significance status. In addition, these results corroborate the importance of regression analyses to evaluate true associations because such analyses minimize the action of confounding variables and thus prevent erroneous conclusions (MEDRONHO et al., 2009). Thus, the multifactorial nature of variations in the population dynamics of parasites, which is expressed as parasite aggregation, becomes evident.

The influence of a fish species' ability to form schools on the degree of parasite aggregation is presented as a determining factor in parasite diversity; however, the various results require additional investigation (MORAND et al., 2000). The formation of schools might be expected to allow greater access of parasitic groups to their hosts because schools increase the size of the resource to be explored from a macroecological perspective, and schooling can influence the abundance of certain parasites, susceptibility of hosts to infection and parasite aggregation values, which was observed in the present study for all parasite taxa except Acanthocephala.

The feeding habits of hosts may also be associated with the degree of parasite aggregation, specifically for endoparasites that are generally transmitted at a trophic level. Because of the amplitude of their trophic spectrum, which is diversified and less specialized than that of fish with more restricted diets (carnivore and planktivore), omnivorous fish would be expected to have access to a greater number and diversity of parasites, which would also influence the degree of aggregation according to diet. However, this behavioral pattern was not observed in the present study, which might have been caused by biological differences in the set of hosts studied in our sample. This explanation can also be applied to differences found in aggregation values, which may be related to different feeding behaviors among fish from different habitats. Benthic fish have a more generalist diet and pelagic fish a more specialized diet; this behavior is consistent with our results, which showed that benthic fish exhibited a greater degree of aggregation in most taxa.

Host size, which is an indirect measure of the degree of susceptibility to parasitic infections, can act as a determinant of variations in the degree of parasite aggregation (POULIN, 2013). However, when analyzing size variations in fish from the studied samples, Poulin (2013) did not observe significant effects on the variability of parasite aggregation, either in the entire sample or within a particular group of parasites. In the present study, however, the host size variable was evaluated as a determinant of parasite aggregation between the sizes of fish from different samples and not within each sample, and the results were significant. This variable initially explains most of the parasite distribution, abundance and diversity in a particular host (LUQUE et al., 2004). The association between the degree of aggregation and body length was expected because hosts with greater length should be able to host a greater number of parasites and because body size is a good measure of total nutrients or energy available in a host to support a parasite species (LUQUE et al., 2004). Therefore, the values of parasite abundance may be higher in hosts with greater total length, thereby increasing the possibility of a higher degree of aggregation.

A similar situation may occur for differences in the degree of aggregation between different parasitic stages (larvae and adults). However, Poulin (2013) did not observe significant effects of the development stage on aggregation levels, which is inconsistent with the results of Lester (2012), who associated the discrepancies with differences in sample size and number of species, which were higher in his study, and a more rigorous assessment of species-specific effects. In the present study, aggregations significantly increased with increases in the proportion of larvae, which indicates that the development stage is a determinant of aggregation. Because of different levels of host specificity, which is greater in larvae than in adults, a greater degree of aggregation was expected for parasites in the larval stage.

In the present study, the factors that could be involved in aggregation were evaluated for each higher taxon, whereas Poulin (2013) tested the development stage and taxon as possible determinants of aggregation. Furthermore, the characteristics of the databases used in the analyses are quite different. Thus, methodological issues may partly explain the different results observed among studies. Moreover, different results may also be a result of the biological characteristics of each parasite species because aggregation as a function of the development stage can be influenced by the particular characteristics of the different biological cycles. Therefore, a comparative analysis by parasite species and an analysis by higher taxa would be required to clarify this issue. 
Regarding host gender and its possible association with the degree of parasite aggregation, the results are heterogeneous, and there are no clearly defined standards that could be attributed to differences in biological and behavioral aspects between male and female hosts. Because there are no studies on the biology and population dynamics of most hosts, it is difficult to establish whether these differences may be true standards in the distribution of parasites or just stochastic results as discussed in studies on the ecology of parasitic communities of marine fish (LUQUE et al., 1996; LUQUE \& ALVES, 2001).

Except for the body size and development stage, the tested variables showed a significance degree of the taxon dependence. On a larger scale, other factors may influence the aggregation of fish parasites in the region because the correlation coefficients for all taxa and stages were lower than 60\%. Macroecological changes may influence parasite abundance (LUQUE \& POULIN, 2008), and according to Braga (2001), the coastal area of Rio de Janeiro is strongly influenced by upwelling systems and subtropical convergence, which are important for the feeding and reproduction of marine organisms (ODEBRECHT \& CASTELLO, 2001). This oceanographic transition zone may represent the northern limit of species from cooler regions or the southern limit for more tropical species, and this zone also receives migratory birds and aquatic mammals from the two hemispheres that may act as definitive hosts of several parasite species of marine fish. The differences between the degrees of parasite aggregation of freshwater and marine fish also reinforce the possibility of macroecological influences on aggregation. Although Marcogliese \& Cone (1997) and Luque $\&$ Poulin (2008) did not detect differences between the parasite diversity of marine and freshwater fish, the possibility that different environmental conditions may influence the degree of aggregation cannot be discounted.

Although parasite aggregation is considered an intrinsic property of parasitic processes, the results of the present study highlight the need to expand investigations by using more comprehensive databases to analyze the combined action of factors with a significant potential influence on the degrees of aggregation and evaluate the role of these factors in the transmission of parasitic diseases.

\section{Acknowledgements}

The authors would like to thank the National Council for Scientific and Technological Development (Conselho Nacional de Desenvolvimento Cientifico e Tecnológico - CNPq) for the financial support provided as a doctoral grant to the first author and a research fellowship to José Luis Luque.

\section{References}

Anderson RM, Gordon DM. Processes influencing the distribution of parasite numbers within host populations with special emphasis on parasite-induced host mortalities. Parasitology 1982; 85(Pt 2): 373-398. http://dx.doi.org/10.1017/S0031182000055347. PMid:7145478

Azevedo RK, Abdallah VD, Luque JL. Biodiversity of fish parasites from Guandu River, southeastern Brazil: an ecological approach. Neotrop Helminthol 2011; 5(2): 185-199.
Braga AC. O ambiente e a biodiversidade. In: Bizerril CRSF, Costa PAS. Peixes Marinhos do Estado do Rio de Janeiro. Rio de Janeiro: FEMAR; 2001. p. 13-32.

Crofton HD. A quantitative approach to parasitism. Parasitology 1971; 62(2): 179-193. http://dx.doi.org/10.1017/S0031182000071420.

Dohoo I, Martin W, Stryhn H. Veterinary epidemiologic research. Charlottetown: University of Prince Edward Island; 2003.

Isaac A, Guidelli GM, Takemoto RM, Pavanelli GC. Prosthenhystera obesa (Digenea), parasite of Salminus maxillosus (Characidae) of the floodplain of the upper Paraná River, Paraná Brazil: influence of the size and sex of host. Acta Sci 2000; 22(2): 523-526.

Katz MH. Multivariable analysis. a pratical guide for clinicians. Cambridge: Cambridge University; 2006b. http://dx.doi.org/10.1017/ CBO9780511811692.

Katz MH. Study design and statistical analysis. Cambridge: Cambridge University; 2006a. http://dx.doi.org/10.1017/CBO9780511616761.

Lester RJG. Overdispersion in marine fish parasites. J Parasitol 2012; 98(4): 718-721. http://dx.doi.org/10.1645/GE-3017.1. PMid:22390836

Luque JL, Alves DR. Ecologia das comunidades de metazoários parasitos, do xaréu, Caranx hippos (Linnaeus) e do xerelete, Caranx latus Agassiz (Osteichthyes, Carangidae) do litoral do estado do Rio de Janeiro, Brasil. Rev Bras Zool 2001; 18(2): 399-410. http://dx.doi.org/10.1590/S010181752001000200011 .

Luque JL, Amato JFR, Takemoto RM. Comparative analysis of the communities of metazoan parasites of Orthopristis ruber and Haemulon steindachneri (Osteichthyes: Haemulidae) from southeastern Brazilian littoral: I structure and influence of size and sex of hosts. Rev Bras Biol 1996; 56(2): 279-292.

Luque JL, Felizardo NN, Tavares LER. Community ecology of the metazoan parasites of namorado sandperches, Pseudopercis numida Miranda-Ribeiro, 1903 and P. semifasciata Cuvier, 1829 (Perciformes: Pinguipedidae), from the coastal zone of the State of Rio de Janeiro, Brazil. Braz J Biol 2008; 68(2): 269-278. http://dx.doi.org/10.1590/ S1519-69842008000200007. PMid:18660954

Luque JL, Lacerda AC, Lizama MAP, Takemoto RM, Bellay S. Aspectos ecológicos. In: Pavanelli GC, Takemoto RM, Eiras JC. Parasitologia de peixes de água doce do Brasil. Maringa: EDUEM; 2013. p. 67-84.

Luque JL, Mouillot D, Poulin R. Parasite biodiversity and its determinants in coastal marine teleost fishes of Brazil. Parasitology 2004; 128(Pt 6): 671682. http://dx.doi.org/10.1017/S0031182004005050. PMid:15206470

Luque JL, Poulin R. Linking ecology with parasite diversity in Neotropical fishes.J Fish Biol 2008; 72(1): 189-204. http://dx.doi.org/10.1111/j.10958649.2007.01695.x.

Marcogliese DJ, Cone DK. Food webs: a plea for parasites. Trends Ecol Evol 1997; 12(8): 320-325. http://dx.doi.org/10.1016/S01695347(97)01080-X. PMid:21238094

Medronho RA, Bloch KV, Luiz RR, Werneck GL. Epidemiologia. São Paulo: Atheneu; 2009.

Morand S, Cribb TH, Kulbicki M, Rigby MC, Chauvet C, Dufour $\mathrm{V}$, et al. Endoparasite species richness of new caledonian butterfly fishes: host density and diet matter. Parasitology 2000; 121(Pt 1): 65-73. http:// dx.doi.org/10.1017/S0031182099006058. PMid:11085226

Odebrecht C, Castello JP. The convergence ecosystem in the Southwest Atlantic. In: Seeliger U, Kjerfve B. Coastal Marine Ecosystems of Latin 
America. Heidelberg: Springer Verlag; 2001. p. 147-165. http://dx.doi. org/10.1007/978-3-662-04482-7_12.

Poulin R. Are there general laws in parasite ecology? Parasitology 2007a; 134(Pt 6): 763-776. http://dx.doi.org/10.1017/S0031182006002150. PMid:17234043

Poulin R. Explaining variability in parasite aggregation levels among host samples. Parasitology 2013; 140(4): 541-546. http://dx.doi.org/10.1017/ S0031182012002053. PMid:23343821

Poulin R. Parasite aggregation: causes and consequences. In: Poulin R. Evolutionary ecology of parasites. Princeton: Princeton University Press; 2007b. p. 134-159.

R Development Core Team R. A language and environment for statistical computing [online]. Vienna: R Foundation for Statistical Computing; 2014 [cited 2014 Jun 10]. Available from:http://www.R-project.org.
Shaw DJ, Dobson AP. Patterns of macroparasite abundance and aggregation in wildlife populations: a quantitative review. Parasitology 1995; 111(1, Suppl): S111-S127. http://dx.doi.org/10.1017/S0031182000075855. PMid:8632918

Venables WN, Ripley BD. Modern applied statistics with S. 4th ed. Springer; 2002. http://dx.doi.org/10.1007/978-0-387-21706-2.

Von Zuben CJ. Implicaçóes da agregação espacial de parasitas para a dinâmica populacional na interação hospedeiro-parasita. Rev Saude Publica 1997; 31(5): 523-530. http://dx.doi.org/10.1590/S003489101997000600014. PMid:9629733

Wilson K, Bjørnstad ON, Dobson AP, Merler S, Poglayen G, Randolph $\mathrm{SE}$, et al. Heterogeneities in macroparasite infections: patterns and processes. In: Hudson PJ, Rizzoli A, Grenfell BT, Heesterbeek H, Dobson AP. The ecology of wildlife diseases. Oxford: Oxford University Press; 2002. p. 6-44. 